\title{
DWI simulation-assisted Machine Learning models for microstructure estimation
}

\author{
Jonathan Rafael-Patino ${ }^{1}$, Thomas $\mathrm{Yu}^{1}$, Victor Delvigne ${ }^{2}$, Muhamed \\ Barakovic $^{1}$, Marco Pizzolato ${ }^{1}$, Gabriel Girard ${ }^{1,3,4}$, Derek K. Jones ${ }^{5,6}$, Erick J. \\ Canales-Rodríguez ${ }^{1,3,7,8}$, and Jean-Philippe Thiran ${ }^{1,3,9}$ \\ 1 Signal Processing Lab (LTS5), École Polytechnique Fédérale de Lausanne, \\ Lausanne, Switzerland. \\ 2 Université de Mons, Mons, Belgium. \\ 3 Radiology Department, Centre Hospitalier Universitaire Vaudois, Lausanne, \\ Switzerland. \\ 4 Centre d'Imagerie BioMédicale (CIBM), Lausanne, Switzerland. \\ ${ }^{5}$ Cardiff University Brain Research Imaging Centre (CUBRIC), School of \\ Psychology, Cardiff University, Cardiff, United Kingdom. \\ 6 Mary McKillop Institute for Health Research, Australian Catholic University, \\ Melbourne, Australia. \\ 7 FIDMAG Germanes Hospitalàries, Sant Boi de Llobregat, Barcelona, Spain. \\ 8 Mental Health Research Networking Center (CIBERSAM), Madrid, Spain. \\ 9 University of Lausanne, Lausanne, Switzerland.
}

\begin{abstract}
Diffusion MRI (DW-MRI) allows for the detailed exploration of the brain white matter microstructure, with applications in both research and the clinic. However, state-of-the-art methods for microstructure estimation suffer from known limitations, such as the overestimation of the mean axon diameter, and the infeasibility of fitting diameter distributions. In this study, we propose to eschew current modeling-based approaches in favor of a novel, simulation-assisted machine learning approach. In particular, we train machine learning (ML) algorithms on a large dataset of simulated diffusion MRI signals from white matter regions with different axon diameter distributions and packing densities. We show, on synthetic data, that the trained models provide an accurate and efficient estimation of microstructural parameters in-silico and from DW-MRI data with moderately high b-values $\left(4000 \mathrm{~s} / \mathrm{mm}^{2}\right)$. Further, we show, on in-vivo data, that the estimators trained from simulations can provide parameter estimates which are close to the values expected from histology.
\end{abstract}

Keywords: Diffusion MRI $\cdot$ Machine Learning $\cdot$ Monte-Carlo Simulations.

\section{Introduction/Related Work}

Diffusion-Weighted MRI (DW-MRI) provides a non-invasive, in-vivo technique for investigating the micro-anatomy of brain tissue. As the DW-MRI signal de- 
pends on the self-diffusion process of water molecules within the intra- and extraaxonal spaces, it can be used to estimate the local, microstructural properties of cells via inverse modeling. A number of microstructure parameters of the white matter fibres such as the statistical distribution of axon diameters (mean and variance) and orientations, as well as the volume fraction occupied by axons (i.e., fibre density) can be inferred from DW-MRI signals through the inversion of complex, non-linear models that require time consuming optimization algorithms. Some examples of state-of-the-art models are AxCaliber [3] and ActiveAx [1], which are used to estimate the axon diameter distribution, and CHARMED [2] and NODDI [15] to estimate the fibre orientations and their volume fractions. To make the fitting more stable, previous models rely on different approximations that require different assumptions. One common assumption is to model the signal from the white matter by a sum of signals from two independent compartments: the intra- and extra-axonal spaces. Other assumptions include modeling axons as perfect, impermeable cylinders. There are some well-known issues with these model-based approaches, including difficulty in separating signals into compartments, model oversimplification, degeneracy, and instability during fitting $[7,15,14]$.

In this study, we explored the feasibility of bypassing some of the limitations of the current inverse models by developing an emerging approach that is based on using DW-MRI simulations as a tool for performing forward modeling [12], [9]. In our approach, we first created a large and detailed dataset of numerical white matter phantoms with varying geometric properties of interest, such as the mean and standard deviation of axon diameters and the axon density. We then generated the DW-MRI signals of these phantoms using a DW-MRI Monte-Carlo simulator [11]. We trained two different machine learning algorithms, i.e., random forest and multi-layer perceptron, to map the simulated signals with and without handcrafted features, to the microstructural parameters. The learned models were then applied to both synthetic and in-vivo brain data.

\section{Methods}

\subsection{Dataset Generation}

Figure 1 shows the structure of our framework from the dataset generation to the accuracy evaluation. In generating the dataset, we express the DW-MRI signal in the brain white matter (WM) as the sum of the signals from the intra-axonal $S_{\text {int }}$ and extra-axonal $S_{\text {ext }}$ compartments, weighted by their relaxation-weighted volume fractions $i c v f$ and $e c v f=1-i c v f$, where $i c v f+e c v f=1$ :

$$
S=(i c v f) S_{i n t}+(1-i c v f) S_{\text {ext }}
$$

The intra-axonal space represents the axons which we model as straight and parallel cylinders, and the extra-axonal space corresponds to the space outside the axons, including the extra-axonal matrix, glial cells, and cerebrospinal fluid, 
etc. We further assume that within a bundle, the axon diameters follow a Gamma distribution [13], with different bundles having different means and standard deviations.

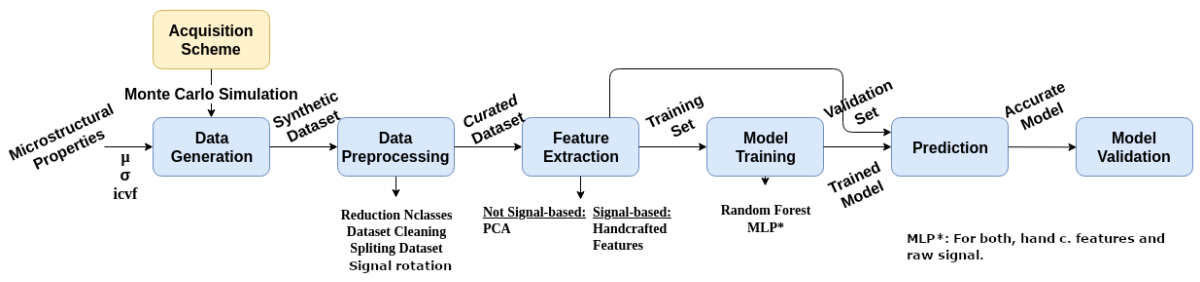

Fig. 1: Framework pipeline

A large dataset of WM phantoms with different geometrical properties was generated by parameterising the intra-axonal space with the volume fraction $i c v f$ as well as the mean $\mu$ and standard deviation $\sigma$ of a Gamma distribution. These parameters were varied according to the realistic values reported in Table 1. A packing algorithm that generates axon geometries matching these predefined parameters was implemented similar to [6]. Then, simulated DWMRI signals for each one of these phantoms were generated using a DW-MRI Monte-Carlo simulator [11] with a specific acquisition protocol consisting of 2 shells with b-values equals to $1000 \mathrm{~s} / \mathrm{mm}^{2}$ and $4000 \mathrm{~s} / \mathrm{mm}^{2} ; \delta=7 \mathrm{~ms}$ and echo time $(\mathrm{TE})=80 \mathrm{~ms}$, with an isotropic voxel resolution of $2 \mathrm{~mm}$. For each shell, the $\Delta$ time was varied as follows, $\Delta=17.3,30,42,55 \mathrm{~ms}$. A total of 30 uniformly sampled directions in the sphere where used per different $\Delta$ time for the shell with b-value $=1000 \mathrm{~s} / \mathrm{mm}^{2}$ and 60 directions for the b-value $=4000 \mathrm{~s} / \mathrm{mm}^{2}$. In addition, to include some uncertainty related to the main fiber orientation, 4 additional signals were generated for each phantom by rotating the original signal by 5 degrees in 4 directions around the mean fiber direction. We chose this dispersion by computing the variance of the main fiber direction in a region of interest in the $\mathrm{CC}$ from the in-vivo data. The resulting substrates were removed if the packed distribution were not close enough to the desired distribution of diameters, or couldn't reach the desired ICVF. Figure 2 shows an example of a generated distribution, as well as the directions used to rotate the phantom's main direction. In total, the final database consisted of 82,400 white matter phantoms and their DW-MRI signals, which were obtained after generating different realizations of each of the 1824 combinations of $i c v f, \mu$ and $\sigma$ (Table1) after pruning.

The in-vivo data were acquired using a 3T Connectome scanner equipped with $300 \mathrm{mT} / \mathrm{m}$ diffusion gradients and using the same imaging parameters employed to generate the synthetic dataset. The same healthy volunteer subject was scanned 5 times to test the robustness of the method to multiple repetitions. 


\begin{tabular}{|l|c|}
\hline Parameters & Values \\
\hline$\mu$ & $\{0.2,0.3,0.5,0.66,0.81,0.97,1.12,1.28,1.44,1.59$, \\
& $1.75,1.91,2.06,2.22,2.34,2.53,2.69,2.84,3\}$ \\
\hline$\sigma$ & $\{0.1,0.3,0.5,1,1.5,2,2.5,3\}$ \\
\hline \multirow{2}{*}{ icvf } & $\{0.35,0.4,0.45,0.5,0.53,0.57$, \\
& $0.6,0.63,0.67,0.7,0.73,0.75\}$ \\
\hline
\end{tabular}

Table 1: Table of values used to generate all the substrates for the mean radius $(\mu)$, the standard deviation of the distribution $(\sigma)$, and the ICVF. A total of 1824 combinations were produced.

In-silico phantom and distribution of diameters
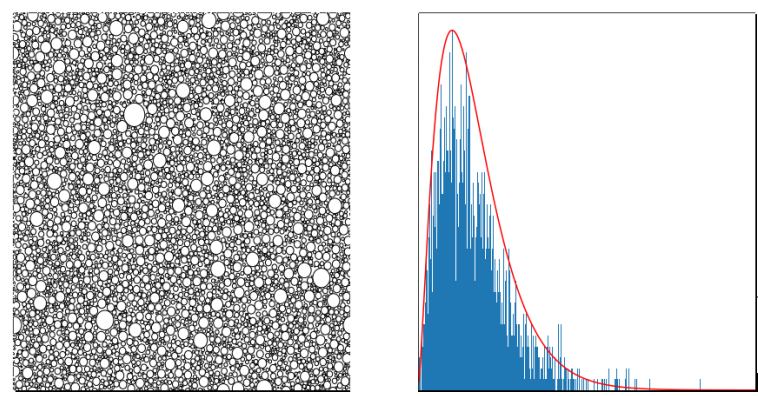

Angular dispersion dirs.

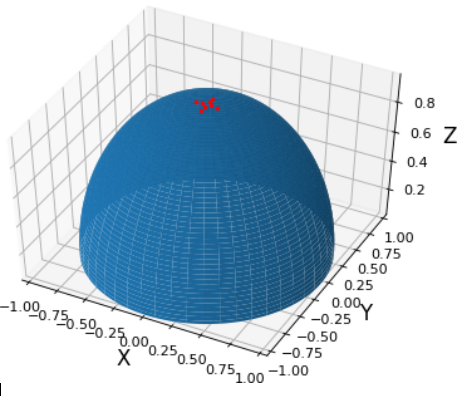

Fig. 2: From left to right, an example of a generated phantom, the resulting distribution of axons after fitting, and the directions used to rotate the main fiber direction to simulate minor angular dispersion.

\subsection{Machine Learning}

To learn the mapping from simulated signals to microstructural parameters, we implemented three different approaches, relying on either handcrafted features or the raw diffusion signal. To create the handcrafted features, the diffusion signal vector was separated into 8 parts, each one corresponding to a different shell with a specific combination of the parameters of the imaging protocol used, i.e., $\Delta, \delta$ and G. Then, for each of these, a Principal Component Analysis was carried out and the first 3 components were selected, which explained more than $98 \%$ of the signal variance. In addition, the fractional anisotropy (FA) and the mean diffusivity (MD) were computed and concatenated to the feature vector. The total feature vector signal consisted of $8 * 3+2=26$ normalized features.

First, we trained a random forest (RF) regressor using the handcrafted features described above. A total of 100 estimator trees with a maximum depth of 17 were used. The random forest parameters were optimized using the Bootstrap aggregation method.

Second, a multi-layer perceptron (MLP) was trained using the same handcrafted features with the following architecture: four dense hidden layers with 
129, 32, 16 and 3 units respectively. We used a rectified linear unit (Relu) as the activation function for all layers, with dropout after the first and second layers with probability 0.01 . The third layer was regularized using $L_{2}$-based kernel and bias regularization with a coefficient of 0.01 . We used the $L_{2}$ loss between the predicted and ground truth parameters as the cost function. Finally, Adam optimizer was employed to train the network for 100 epochs with a batch size of 10. We refer to this method as MLP-feat on the rest of the paper.

Third, we trained an MLP using the DW-MRI signal directly, with no feature extraction. The architecture consists of 6 hidden layers with 400, 200, 100, 56, 16 and 3 neurons respectively. The fifth layer is regularized as in the MLP above. Other training details are identical to those used in the MLP above. We refer to this method as MLP-raw to differentiate it from the previous one trained with handcrafted features.

In this study, $80 \%$ of the dataset was used for training and validation, while $20 \%$ was held-out for testing; 10 -fold cross-validation to mitigate overfitting was performed. The three described approaches were tested on both, the held-out synthetic data and five in-vivo DW-MRI images masked into the CC. As a baseline for the in-vivo data, we compare these results with those of the AMICO [4] implementation of ActiveAx [1], using the default regularization parameters and dictionary.

\section{Results and Discussion}

Figure 3 shows the microstructure parameters estimated by the three ML algorithms from synthetic data. Our main finding is that they are able to accurately estimate both the mean and standard deviation of the axon diameter distributions even for diameters much smaller than those estimated in previous studies (i.e., 2um), and using an acquisition protocol employing b-values lower than the conventional ones used for diameter estimation $[1,4]$. To explain why the proposed ML techniques can improve the estimation of axons with smaller diameters, it is important to remember that the model-based approaches determine the axon diameter entirely from an intra-axonal model, as the signal from the extra-axonal space is difficult to relate analytically to the underlying microstructure parameters. The limited diffusion contrast of the DW-MRI signal from the intra-axonal space along the direction perpendicular to the fibers hampers the estimation of axons with small diameters. In contrast, applying ML techniques to the whole signal allows finding hidden non-trivial and nonlinear relationships between the microstructure parameters and the DW-MRI signal from both the intra- and the extra-axonal spaces. As the properties of the diffusion process in the extra-axonal space (i.e., mean displacement length, tortuosity, time-dependent diffusion) are highly influenced by the microstructure features of the intra-axonal space, the ML algorithms can exploit this additional information. Another important factor is that, by defining a specific distribution of axon diameters in our forward model, the ML algorithms could predict the full 
distribution using only the information from the right-tail of the distribution, which is not affected by the contrast/resolution problem mentioned before.

\begin{tabular}{|l|l|l|}
\hline \multicolumn{3}{|l|}{ Cross validation MAE } \\
\hline RF feat. & MLP feat. & MLP raw \\
\hline 0.028 & 0.070 & 0.134 \\
\hline
\end{tabular}

Table 2: Mean absolute error (MAE) on the cross validation dataset for the three trained classifiers: RF, MLP trained with feature vectors, and MLP trained with the raw signal.

Table 2 shows the mean absolute error of the three parameters estimated on the noise-free test dataset. From Figure 3 and Table 2, we can see that the RF regressor has the best accuracy for all three parameters, with ICVF the most difficult parameter to estimate. Both MLP methods had good accuracy during training but lower accuracy on the test dataset than the RF regressor.

Figure 5 shows the estimated mean diameter $(\mathrm{mD})$ maps for two arbitrarily selected scans from the in-vivo data, in the same anatomical cross-section for the three methods. For each ML regressor, the parameter estimates are quite consistent over the five images used; that is, the values and the anatomical locations of small or large mean diameters are similar across scans. In addition, we show the axon's diameter maps computed with AMICO-ActiveAx, which are notably higher than those from the ML methods. Is important to notice however that the used protocol is far from idoneous for the former methodwhich requires b-values as high as $\mathrm{b}=9,000 \mathrm{~s} / \mathrm{mm}^{2}$ in ex-vivo conditions [5] to estimate mean axon diameters below $2 \mathrm{um}$ - and thus, such over-estimation is expected.

Figure 4 depicts the histograms of the estimated mean diameters. There is a noticeable variability between the parameter estimates of two different regressors. This is important as this lack of consensus between regressors implies that at least one must be biased and shows how three different estimators, with similar performance and accuracy in in-silico data, can estimate remarkably different distributions in in-vivo data. This is likely to be and effect of several compartments not included in our training data: axonal tortuosity and diameter changes, or even T2 relaxation or artifacts effects. Therefore, this study will benefit of including such effects during the training. Or the use of more advanced machine learning models [10]. Notably however, while all ML models predicted mean diameters with magnitudes close - but still higher - from the ones extracted from histology, the trend in mean diameter on the genu, truncus and splenium of the $\mathrm{CC}$ predicted by the RF regressor (i.e., the optimal one in synthetic data) matches that from a previous study on electron-microscopy for the distribution of axon diameters in cortical white matter [8]. 


\section{Regressor Parameter Estimates}

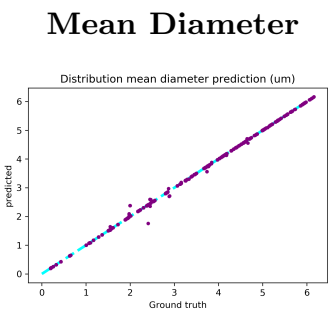

ICVF
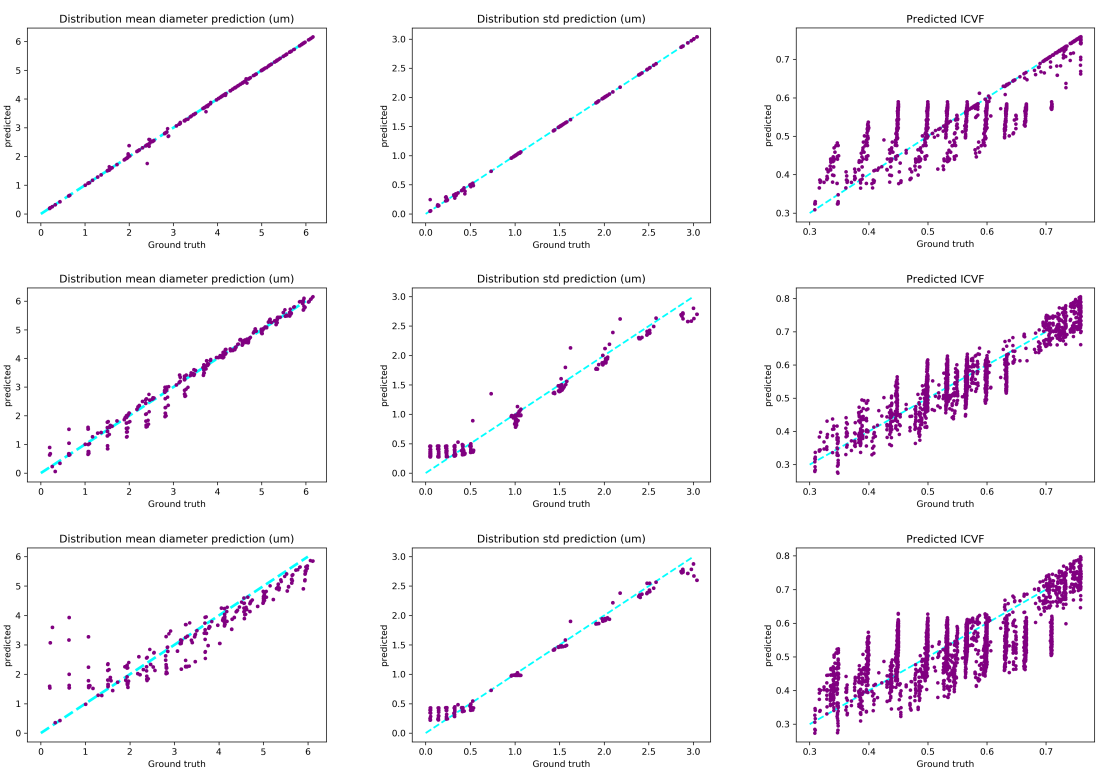

Fig. 3: Estimates of the trained regressors on the validation dataset. From top to bottom, results for the Random Forest (top), MLP trained with hand crafted features (middle row), and the MLP trained with the raw signal (bottom). From left to right, ground truth vs the estimated parameters on the validation dataset for the mean diameter, the standard deviation, and the ICVF respectively.

\section{Conclusions}

The main focus of this work is the introduction of a emerging simulation-based technique for the microstructural parameter estimation; from the construction of a large dataset of realistic numerical phantoms to training machine learning algorithms on the corresponding simulated signals. A comprehensive dataset accounting for a wide variety of parameters characterizing axon packing and size, as well as small angular dispersion mimicking that which occurs in the CC was generated. We demonstrated that machine learning models with and without handcrafted features can accurately recover the mean and standard deviation of the axon diameter distribution on synthetic data and from DW-MRI data with moderately high b-values $\left(4000 \mathrm{~s} / \mathrm{mm}^{2}\right)$. The proposed approach allowed us to estimate, for the first time, the number-weighted distribution of axon diameters, which cannot be estimated with previous DW-MRI modeling techniques. As this is the distribution conventionally reported in histological studies, this study may help to fill the gap between in-vivo DW-MRI and postmortem histol- 
CC estimated mean diameters

$\mathrm{RF}$ regressor

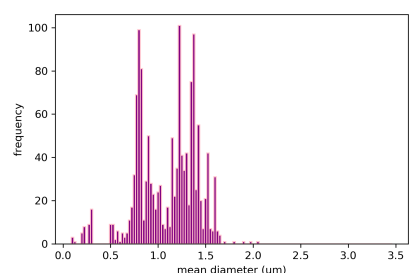

MLP feat.

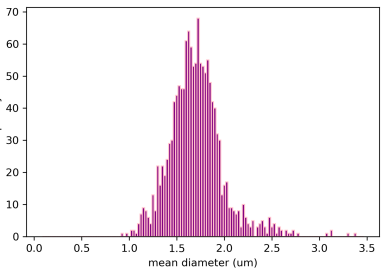

MLP raw

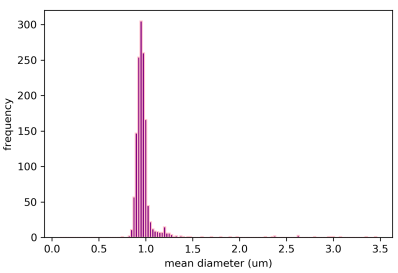

Fig. 4: Joint histogram of the estimated mean diameters in the segmented mask of the CC across 5 scans for (from left to right) the RF regressor, MLP-feat and the MLP-raw. Each histogram was computed using the combined estimates of the 5 in-vivo scans.

ogy. Furthermore, once trained, machine learning models require a few seconds of computation time for estimating the microstructure parameters in the wholebrain white matter. Finally, the in-vivo results show consistent trends and values for all 5 subject scans with the same ML model, however, there are qualitative differences between the different models that should be explored in future studies. Our findings show that values reported in the CC for all ML models are close to those found in histology, with the RF regressor further replicating the expected spatial trend in mean diameter [8]. It is important to mention, however, that in this work a simplified model of the CC microstructure is employed by considering a 2 compartment model which assumes that axons are completely straight cylinders, without micro-dispersion along the axons. Nevertheless, since it is straightforward to generate more realistic phantoms (e.g. axons with undulations and angular dispersion) and simulate the corresponding DW-MRI signals using state-of-the-art MC simulators, both limitations can be addressed in the future by generating more complex axon configurations without significantly modifying the machine learning models and related parameters. Future in-silico validations will be conducted to evaluate the robustness of the method as a function of the signal-to-noise ratio and acquisition protocol.

\section{Acknowledgements}

This work was supported by EPFL through the use of the facilities of its Scientific IT and Application Support Center.

We gratefully acknowledge the support of NVIDIA Corporation with the donation of the Titan Xp GPU used for this research. 


\section{In-vivo Random forest $m D$ predictions}

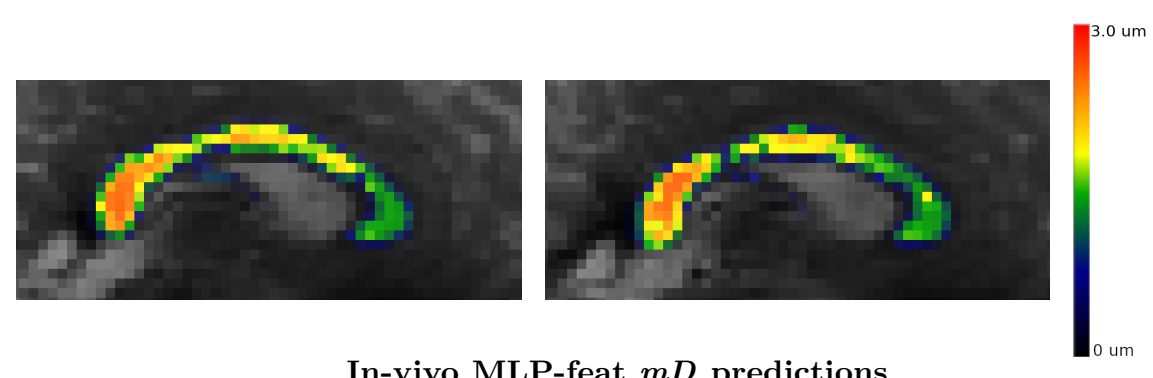

In-vivo MLP-feat $m D$ predictions
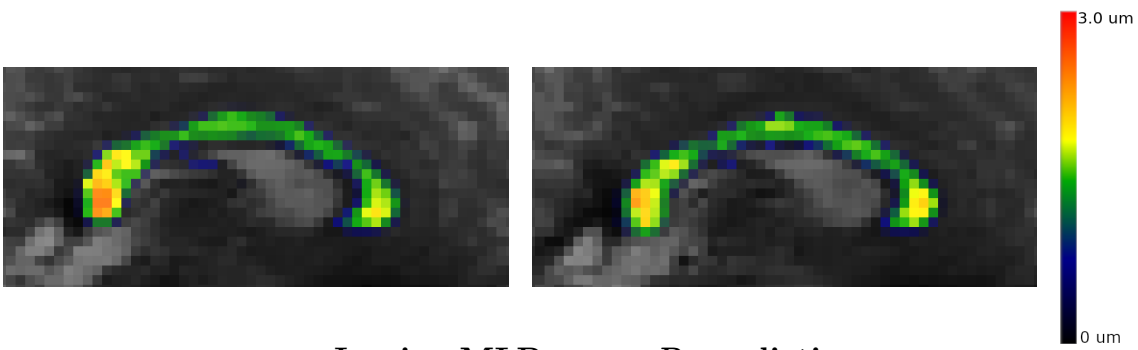

In-vivo MLP-raw $m D$ predictions
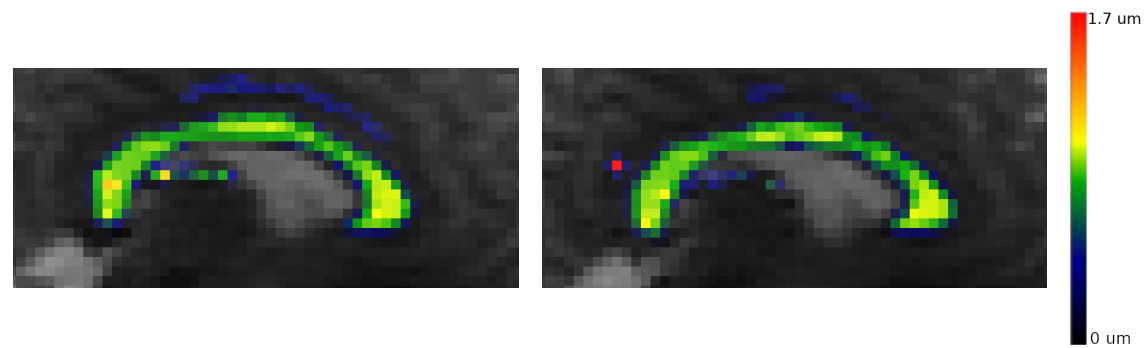

In-vivo AMICO-ActiveAx $m D$ predictions
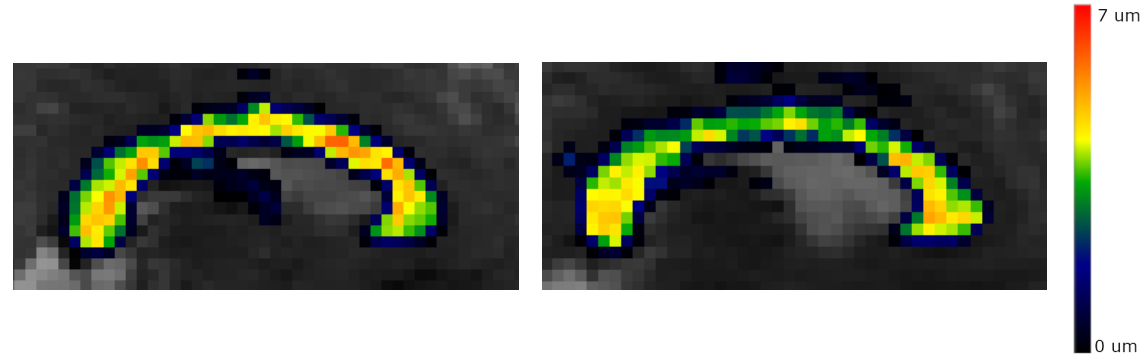

Fig. 5: Midsagittal plane of the estimation maps for two subjects (showed from posterior to anterior). The colorbars where adjusted per model to highlight the regions with higher and lower values. In one of the MLP-raw predictions it can be notice the presence of a notorious outlayer values in regions voxels outside the CC. 


\section{References}

1. Alexander, D.C., Hubbard, P.L., Hall, M.G., Moore, E.A., Ptito, M., Parker, G.J., Dyrby, T.B.: Orientationally invariant indices of axon diameter and density from diffusion MRI. NeuroImage (2010). https://doi.org/10.1016/j.neuroimage.2010.05.043

2. Assaf, Y., Basser, P.J.: Composite hindered and restricted model of diffusion (CHARMED) MR imaging of the human brain. NeuroImage (2005). https://doi.org/10.1016/j.neuroimage.2005.03.042

3. Assaf, Y., Blumenfeld-Katzir, T., Yovel, Y., Basser, P.J.: AxCaliber: A method for measuring axon diameter distribution from diffusion MRI. Magnetic Resonance in Medicine (2008). https://doi.org/10.1002/mrm.21577

4. Daducci, A., Canales-Rodríguez, E.J., Zhang, H., Dyrby, T.B., Alexander, D.C., Thiran, J.P.: Accelerated Microstructure Imaging via Convex Optimization (AMICO) from diffusion MRI data. NeuroImage (2015). https://doi.org/10.1016/j.neuroimage.2014.10.026

5. Dyrby, T.B., Sagaard, L.V., Hall, M.G., Ptito, M., Alexander, D.C.: Contrast and stability of the axon diameter index from microstructure imaging with diffusion mri. Magnetic Resonance in Medicine 70(3), 711 (2013), 10.1002/mrm.24501

6. Hall, M.G., Alexander, D.C.: Convergence and parameter choice for monte-carlo simulations of diffusion mri. IEEE Transactions on Medical Imaging 28(9), 13541364 (Sep 2009). https://doi.org/10.1109/TMI.2009.2015756

7. Jelescu, I.O., Veraart, J., Fieremans, E., Novikov, D.S.: Degeneracy in model parameter estimation for multi-compartmental diffusion in neuronal tissue. NMR in Biomedicine 29(1), 33-47 (2016). https://doi.org/10.1002/nbm.3450, https://onlinelibrary.wiley.com/doi/abs/10.1002/nbm.3450

8. Liewald, D., Miller, R., Logothetis, N., Wagner, H.J., Schüz, A.: Distribution of axon diameters in cortical white matter: an electron-microscopic study on three human brains and a macaque. Biological Cybernetics (2014). https://doi.org/10.1007/s00422-014-0626-2

9. Nedjati-Gilani, G.L., Schneider, T., Hall, M.G., Cawley, N., Hill, I., Ciccarelli, O., Drobnjak, I., Wheeler-Kingshott, C.A., Alexander, D.C.: Machine learning based compartment models with permeability for white matter microstructure imaging. NeuroImage 150(July 2016), 119-135 (2017). https://doi.org/10.1016/j.neuroimage.2017.02.013, http://dx.doi.org/10.1016/j.neuroimage.2017.02.013

10. Rafael-Patino, J., Barakovic, M., Girard, G., Daducci, A., Thiran, J.P.: Learning global brain microstructure maps using trainable sparse encoders (2019), http://infoscience.epfl.ch/record/265398

11. Rafael-Patino, J., Romascano, D., Ramirez-Manzanares, A., Canales-Rodríguez, E.J., Girard, G., Thiran, J.P.: Robust Monte-Carlo Simulations in Diffusion-MRI: Effect of the substrate complexity and parameter choice on the reproducibility of results (aug 2019), http://arxiv.org/abs/1908.11203

12. Rensonnet, G., Scherrer, B., Girard, G., Jankovski, A., Warfield, S.K., Macq, B., Thiran, J.P., Taquet, M.: Towards microstructure fingerprinting: Estimation of tissue properties from a dictionary of monte carlo diffusion mri simulations. NeuroImage 184, $964 \quad-980$ (2019). https://doi.org/https://doi.org/10.1016/j.neuroimage.2018.09.076, http://www.sciencedirect.com/science/article/pii/S1053811918319487 
13. Sepehrband, F., Alexander, D.C., Clark, K.A., Kurniawan, N.D., Yang, Z., Reutens, D.C.: Parametric Probability Distribution Functions for Axon Diameters of Corpus Callosum. Frontiers in Neuroanatomy (2016). https://doi.org/10.3389/fnana.2016.00059

14. Yu, T., Pizzolato, M., Girard, G., Rafael-Patino, J., Canales-Rodríguez, E.J., Thiran, J.P.: Robust biophysical parameter estimation with a neural network enhanced hamiltonian markov chain monte carlo sampler. In: Chung, A.C.S., Gee, J.C., Yushkevich, P.A., Bao, S. (eds.) Information Processing in Medical Imaging. pp. 818-829. Springer International Publishing, Cham (2019)

15. Zhang, H., Schneider, T., Wheeler-Kingshott, C.A., Alexander, D.C.: NODDI: Practical in vivo neurite orientation dispersion and density imaging of the human brain. NeuroImage 61(4), 1000-1016 (mar 2012) 